\title{
Seismology of quiescent coronal loops
}

\author{
Y. Taroyan and R. Erdélyi \\ Solar Physics and upper-Atmosphere Research Group, \\ Department of Applied Mathematics, University of Sheffield, Sheffield, S3 7RH, UK \\ email: y.taroyan@sheffield.ac.uk, robertus@sheffield.ac.uk
}

\begin{abstract}
The determination of the physical parameters of coronal loops remains both an observational and a theoretical challenge. A new diagnostic technique for quiescent dynamically heated coronal loops, based on the analysis of the power spectra of Doppler shift time series, is proposed. It is assumed that a given loop is heated randomly both in space and time by small-scale discrete impulsive events of unspecified nature. It is shown here that, depending on the heliographic position of the loop and the orientation of the observing instrument, various harmonics can be identified in the power spectra of line shift time series. The frequency peaks are sensitive to changes in the average loop temperature and are proposed to use as a temperature diagnostic tool. The analysis of the power spectra also allows to distinguish uniformly heated loops from loops heated near their footpoints.
\end{abstract}

Keywords. Sun: corona, Sun: oscillations, line: profiles, hydrodynamics

\section{Introduction}

The study of magnetic loops is crucial to our understanding of the dynamics of solar coronal plasma. A vast number of new approaches have attempted to compare theoretical models with loop observations. Such comparisons have been carried out mostly by measuring the temperature and density profiles using line intensity ratios from spectrometers (Brković et al. 2002; Landi \& Landini 2004), differential emission measure analysis (Schmelz et al. 2002; Martens et al. 2002) or filter ratios from imagers. A successful comparison of measured and observed temperature profiles could in principle be a diagnostic tool for loop heating. However, temperature measurements have been the subject of a heated debate. Some authors have questioned the use of these methods (see, e.g., Testa et al. 2002; Chae et al. 2002). The measurements are often ambiguous and contradictory.

In this respect, the newly emerging field of coronal seismology has a great potential which has not yet been fully exploited. Studies indicate that both wave and reconnection driven heating appear in small scale bursts of energy which are randomly distributed along the loops (see, e.g., Moriyasu et al. 2004; Einaudi \& Velli 1999). We are mainly interested in analysing the observational consequencies of such localised heating events without specifying the exact nature of the heating process. Recently Taroyan et al. (2006) found that the random nanoflare heating model is able to qualitatively reproduce the observed dynamics and the average red-shifts typical for the lines covering the upper transition region and lower corona. The present short paper investigates the possibility of applying the helioseismic approach of power spectrum analysis to coronal loops: Doppler shift time series are Fourier analysed and the resulting power spectra are expected to show the presence of discrete peaks corresponding to standing wave harmonics. This is a simple manifestation of the superposition principle, i.e., any linear perturbation of a physical system is a superposition of its normal modes. We mainly address the feasibility and the efficiency of the proposed new method. 

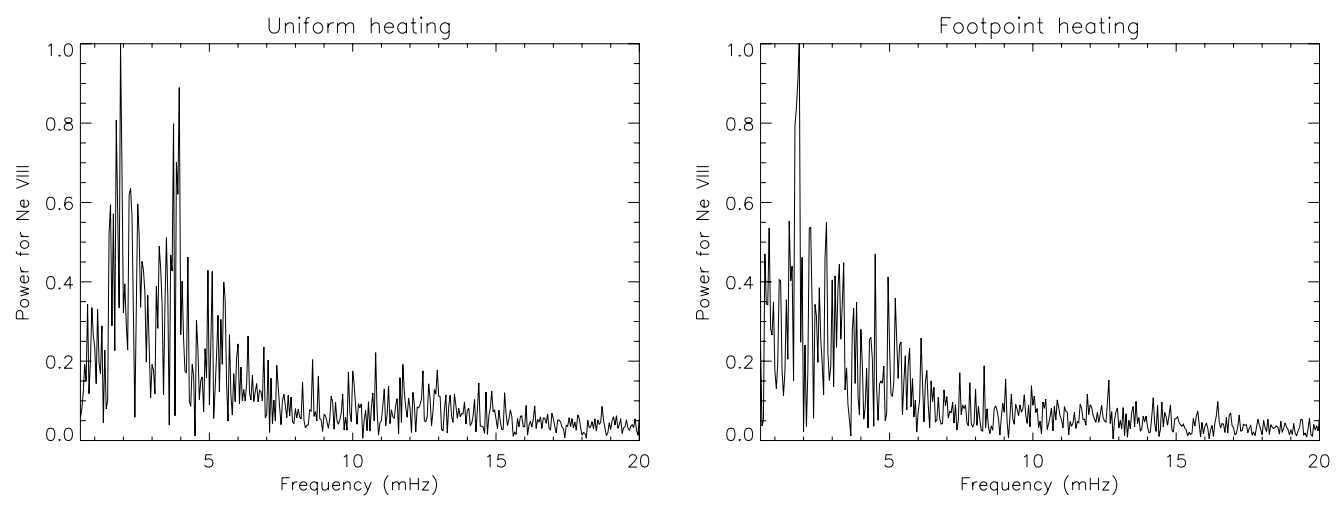

Figure 1. Normalised power spectra for the time series of the Doppler shift of the Ne VIII resonance line. Two different cases corresponding to uniform and footpoint heating are presented.

\section{Results}

A $40 \mathrm{Mm}$ long loop is randomly heated by small-scale bursts of energy which are randomly distributed in time. The total duration of the simulations is $20,000 \mathrm{~s}$. The loop is maintained at an average temperature of $1 \mathrm{MK}$. Two different types of heating are considered: uniform and footpoint heating. In the first case, the heating events randomly occur along the entire length of the loop. In the second case, the heating events are mainly concentrated near the footpoints of the loop. The line profiles for the Ne VIII resonant line are synthesized. The corresponding time series for the Doppler shift are Fourier analysed. The resulting power spectra for the two different cases are plotted in Figure 1. It is assumed that in both cases the loop is located on the disc centre. The peaks at around $2 \mathrm{mHz}$, seen in both panels of Figure 1, represent the frequencies of the fundamental mode. These peaks can be used to determine the average temperarture of the loop. The second peak representing the first harmonic is only seen in the left panel of Figure 1. The absence of the corresponding peak in the right panel is due to increased conduction near the footpoints in the case of footpoint heating. This in turn leads to stronger damping of the oscillations where the maxima in the amplitudes of the first harmonic occur. Therefore, by analysing the power spectra of Doppler shift time series one can distinguish uniformly heated loops from loops heated near their footpoints.

\section{Acknowledgements}

YT is grateful to PPARC for financial support. RE acknowledges M. Kéray for patient encouragement, and NSF, Hungary (OTKA. Ref No. TO43741).

\section{References}

Brković, A., Landi, E., Landini, M., Redi, I., \& Solanki, S. 2002, A\& A 383, 661

Chae, J., Park, Y. D., Moon, Y.-J., Wang, H., \& Yun, H. S. 2002, ApJ 567, L159

Einaudi, G., Velli, M. 1999, Phys. Plasmas 6, 4146

Landi, E., \& Landini, M. 2004, ApJ 608, 1133

Martens, P. C. H., Cirtain, J. W., \& Schmelz, J. T. 2002, ApJ 577, L115

Moriyasu, S., Kudoh, T., Yokoyama, T., \& Shibata, K. 2004, ApJ, 601, L107

Schmelz, J. T., Scopes, R. T., \& Cirtain, J. W. 2002, Adv. Space Res. 30, 507

Taroyan Y., Bradshaw, S. J., \& Doyle, G. 2006, A\&A 446, 315

Testa, P., Peres, G., Reale, F., \& Orlando, S. 2002, ApJ 580, 1159 